Gut and Liver, Vol. 10, No. 4, July 2016, pp. 509-519

\title{
Evaluation and Management of Hepatic Encephalopathy: Current Status and Future Directions
}

\author{
Duminda Suraweera ${ }^{1}$, Vinay Sundaram ${ }^{2}$, and Sammy Saab ${ }^{3,4}$ \\ ${ }^{1}$ Department of Medicine, Olive View Medical Center, Sylmar, ${ }^{2}$ Department of Medicine, Cedars-Sinai Medical Center, Los Angeles, Departments \\ of ${ }^{3}$ Medicine and ${ }^{4}$ Surgery, University of California at Los Angeles, Los Angeles, CA, USA
}

Hepatic encephalopathy is a spectrum of neurocognitive manifestations often seen in patients with liver injury or rarely in patients with portosystemic shunting without liver injury. It can be divided into minimal (covert) hepatic encephalopathy and overt hepatic encephalopathy, depending on the severity. Patients with hepatic encephalopathy have compromised clinical outcomes, decreased quality of life, and increased healthcare utilization, often resulting in a heavy financial and personal burden on caregivers. The diagnosis remains largely clinical, with the exclusion of possible other causes for the altered mental status. Current treatment strategies include nonabsorbable disaccharides and antibiotics. This review will focus on the diagnosis, management and clinical impact of hepatic encephalopathy. (Gut Liver 2016;10:509-519)

Key Words: Hepatic encephalopathy; Liver cirrhosis; Liver diseases; Minimal hepatic encephalopathy

\section{INTRODUCTION}

Hepatic encephalopathy (HE) is often a serious sequela of chronic liver disease with significant morbidity, mortality and healthcare costs. In the United States HE accounts for 22,931 hospitalizations with an average stay of 8.5 days and a total cost of $\$ 64,108$ per case. ${ }^{1}$ HE can be graded by severity using the West Haven Criteria and can be divided into minimal HE (MHE) and overt HE (OHE) (Fig. 1). While the basis of HE is likely multifactorial, increased systemic circulation of ammonia plays a pivotal role. Thus current management of HE focuses on reducing blood ammonia concentrations using nonabsorbable disaccharides and antibiotics. Definitive treatment of manifestations of portal hypertension such as HE requires liver transplant. Hepatic encephalopathy is not only associated with poor prog- nosis but also significant detrimental effects to quality of life and a substantial burden to caregivers and healthcare systems.

\section{PATHOGENESIS}

The pathogenesis of HE is likely multifactorial. Ammonia and dysregulation of the urea cycle is often implicated in the pathogenesis of HE. Nitrogenous compounds excreted by gut bacteria are transported to the liver via the portal circulation where it, along with endogenous nitrogen, enter the urea cycle. The end process is the generation of urea which is subsequently excreted through urine. ${ }^{2}$ In advanced liver disease, damaged hepatocytes and the development of portosystemic shunts results in ammonia bypassing the liver and accumulating in the systemic circulation (Fig. 2). ${ }^{3}$ Ammonia then crosses the blood-brain battier and is metabolized by astrocytes to synthesize glutamine from glutamate via glutamine synthetase. ${ }^{4}$ Glutamine increases the osmotic pressure within the astrocyte resulting in morphologic malformations similar to those seen in Alzheimer's disease Type II..$^{5-7}$ In addition astrocyte swelling stimulates the formation of reactive oxygen species that further increases astrocyte swelling. ${ }^{8}$ It is believed that the glutamine produced from ammonia is transported from the cytoplasm to mitochondria where it is again converted back to glutamate and ammonia. This results in mitochondrial dysfunction, increasing production of reactive oxygen species. ${ }^{9}$ Evaluation of cortical brain tissue postmortem have found higher levels of RNA oxidation markers in patients with HE than those without liver disease. ${ }^{10}$ Studies have also shown an association between hyperammonemia and increased gamma-amino-n-butyric acid (GABA). ${ }^{11,12}$ Possible mechanisms for this include direct action of ammonia on GABA receptors and inhibition of astrocyte uptake of GABA thereby resulting in increased levels of extracellular GABA. ${ }^{13}$ Portosystemic shunts

Correspondence to: Sammy Saab

Pfleger Liver Institute, UCLA Medical Center, 200 Medical Plaza, Suite 214, Los Angeles, CA 90095, USA

Tel: +1-310-206-6705, Fax: +1-310-206-4197, E-mail: SSaab@mednet.ucla.edu

Received on August 27, 2015. Accepted on August 31, 2015.

pISSN 1976-2283 eISSN 2005-1212 http://dx.doi.org/10.5009/gnl15419

@ This is an Open Access article distributed under the terms of the Creative Commons Attribution Non-Commercial License (http://creativecommons.org/licenses/by-nc/4.0) which permits unrestricted non-commercial use, distribution, and reproduction in any medium, provided the original work is properly cited. 


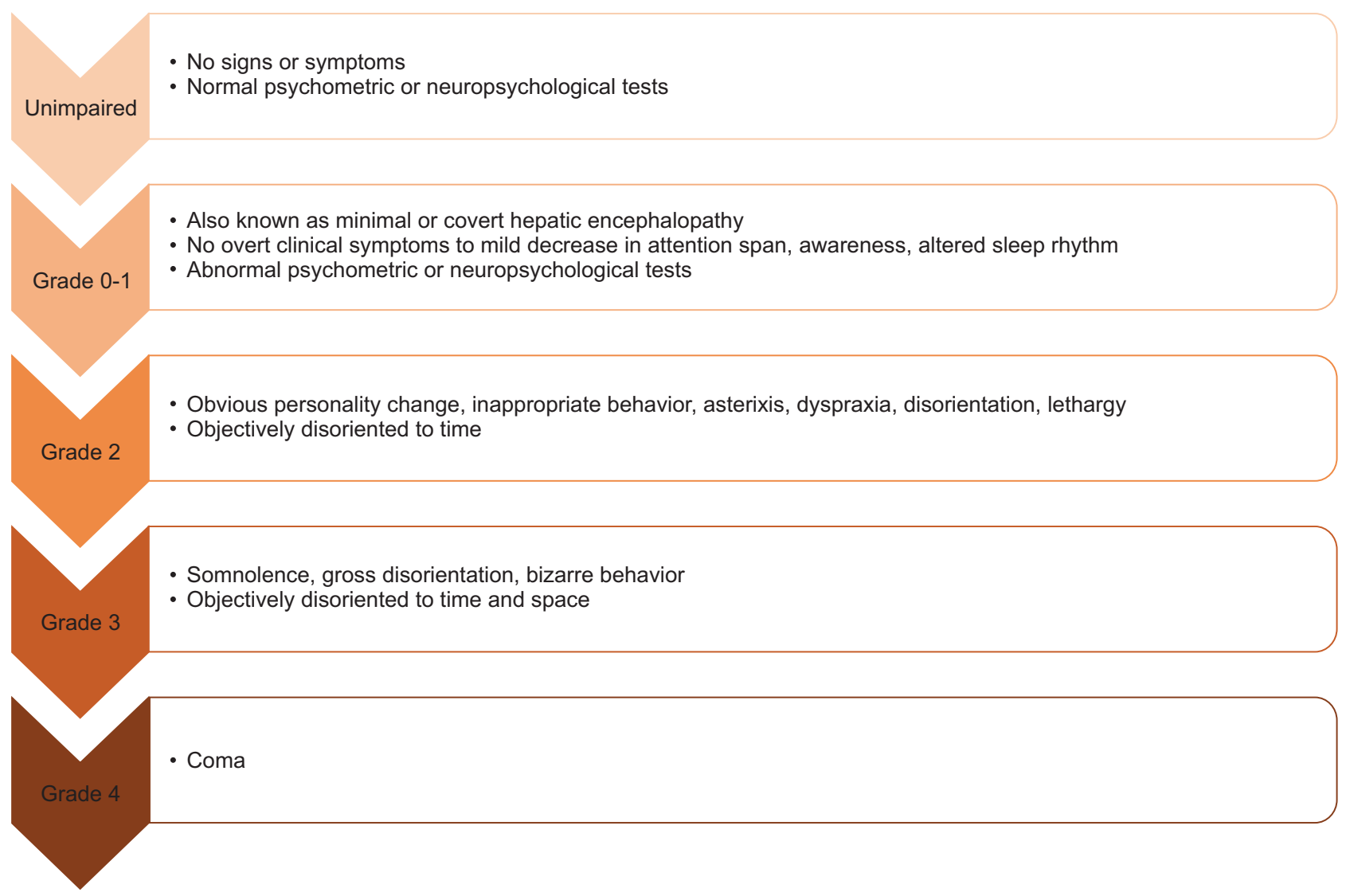

Fig. 1. West Haven Criteria classification of hepatic encephalopathy.

Organism

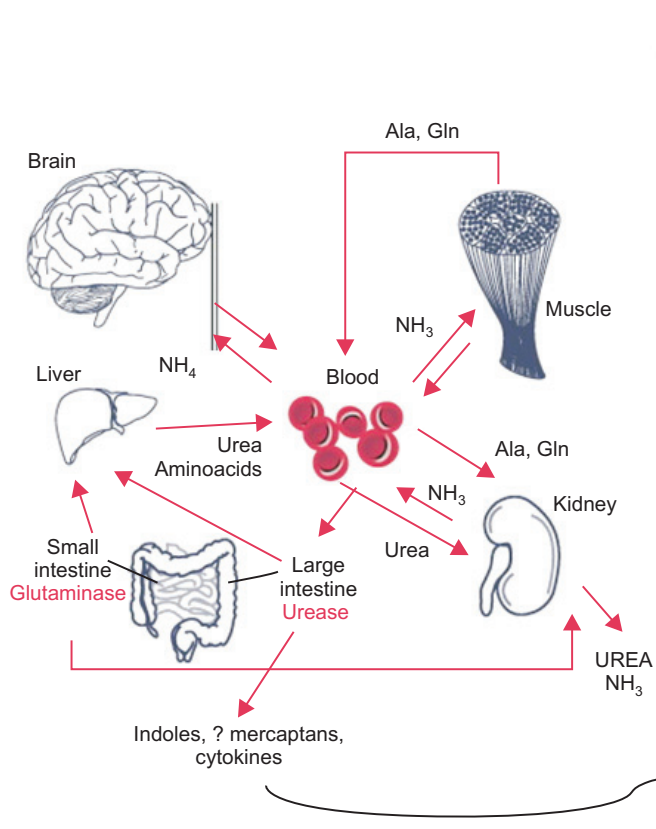

Organ

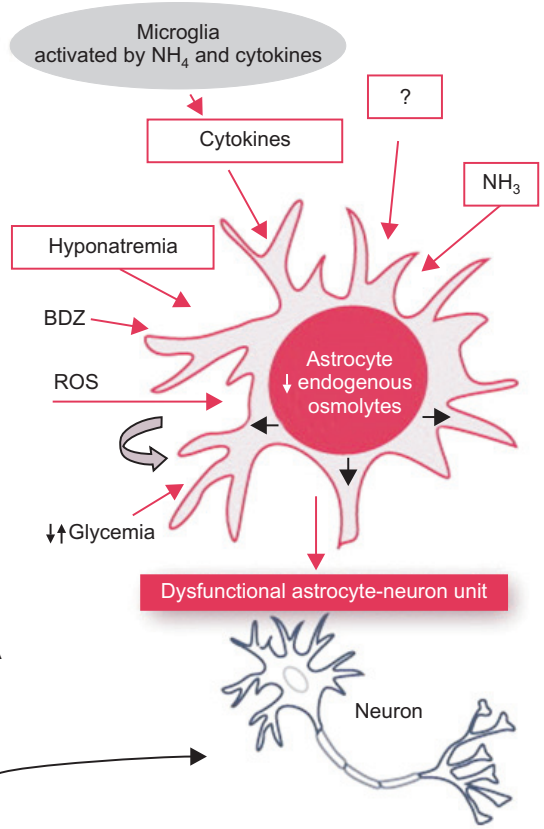

Fig. 2. Ammonia imbalance leading to astrocyte swelling that subsequently results in astrocyte-neuron dysfunction. Adapted from Amodio P. Hepatic encephalopathy. In: Lee SS, Moreau R, eds. Cirrhosis: a practical guide to management. Chichester: John Wiley \& Sons, 2015, with permission from John Wiley \& Sons. ${ }^{100}$ 
can also cause HE by diverting venous blood from the portal system into systemic circulation. These shunts often develop in the setting of advanced liver disease but can also be present in the absence of liver disease. Shunts may be iatrogenic with the placement of transjugular intrahepatic portosystemic shunt (TIPS). Often these patients experience an increase in the frequency of $\mathrm{HE}$ post procedure and a decrease in HE episodes after occlusion of these shunts. ${ }^{14}$

\section{PRESENTATION AND DIAGNOSIS}

\section{Clinical presentation}

Hepatic encephalopathy can present with a wide spectrum of neurocognitive manifestations. In the case of MHE, there may not be any obvious clinical changes. However these patients have abnormal psychometric tests and subtle changes in personality may be reported by caregivers. ${ }^{15}$ As progression to OHE occurs patients will have greater disturbances in cognition leading to more drastic personality changes, irritability and disinhibition. Motor abnormalities such as hypertonia and hyperreflexia as well as extrapyramidal dysfunction such as rigidity, bradykinesia, dyskinesia, hypokinesia, and slowness of speech are also often present. ${ }^{16}$ Sleep disturbances with excessive daytime sleepiness are another common manifestation in OHE. ${ }^{17}$ While asterixis is not specific to HE, it is an important finding in HE. The International Society of Hepatic Encephalopathy and Nitrogen Metabolism recommends that the onset of asterixis and disorientation be used to define OHE. ${ }^{18}$ While historically it has been thought that manifestations of HE are completely reversible, recent research and clinical experience point towards persistent and cumulative deficits in response inhibition, working memory and learning. ${ }^{1920}$

\section{Diagnosis of minimal hepatic encephalopathy}

MHE is defined as patients with abnormal psychometrics test without meeting criteria for OHE. Psychometric Hepatic Encephalopathy Score (PHES) has been shown to be specific and sensitive in determining MHE. ${ }^{17}$ PHES is composed of five tests: number connection test-A, number connection test-B, serial dotting test, line tracing test, and digit symbol test. An alternative is using these neuropsychological tests individually. Several computerized tests have also aided in the diagnosis of MHE. Inhibitory control testing uses letters projected on the screen to test if patients can respond only to certain letters. Patients with MHE tend to have longer reaction times, high rates of inappropriate reactions and lower rates of appropriate reactions. The inhibitory control test has a sensitive of $87 \%$ and specificity of $77 \%{ }^{21}$ Cognitive Drug Research test battery is yet another tool that has been shown to be comparable to PHES in the diagnosis of MHE. ${ }^{22}$ This particular tests evaluates multiple cognitive domains including power of attention, continuity of attention, quality of episodic and working memory as well as the speed of memory. EncephalApp is a smartphone application that measures the time it takes patients to correctly identify a series of symbols and words with varying colors. In a recent validation study the EncephalApp correctly identified MHE in all 167 patients that were tested. ${ }^{23}$ Neurophysiological testing can also be used to detect impairments seen in MHE. Clicker flicker frequency (CFF) is simple test that asks the patient to identify when a light begins to flicker. Initially the light pulse is set at a frequency of $60 \mathrm{~Hz}$ and is reduced incrementally at $0.1 \mathrm{~Hz}$ per second. The test is considered positive if the patient identifies flickering bellow $39 \mathrm{~Hz}$. CFF has a sensitivity of 65\% and a specificity of $91 \%$ in diagnosis MHE. ${ }^{24}$ Electroencephalography (EEG) can be used however its sensitivity varies, ranging from $43 \%$ to $100 \% .^{25}$ Increased variability and resource requirements make EEG less than ideal in the diagnosis of MHE. Similarly evoked potentials can be used but the results are inconsistent. ${ }^{21}$ While the West Haven Criteria (WHC) has been classically used for grading OHE, the International Society for Hepatic Encephalopathy and Nitrogen Metabolism (ISHEN) recommends that WHC grade 1 be classified as covert hepatic encephalopathy. ${ }^{26}$

\section{Diagnosis of overt hepatic encephalopathy}

Overt hepatic encephalopathy may be precipitated by an event such as electrolyte imbalance, gastrointestinal bleeding or acute liver injury. Initial evaluation should include investigation into possible instigating factors. In addition OHE should be differentiated from stroke or other causes of neuralgic disturbance that can mimic OHE. The West Haven Criteria is most often used to grade OHE (Fig. 1). ${ }^{26,27}$ This criteria grades the neurological deficits seen in OHE patients, including motor system abnormalities and behavioral or personality changes (Fig. 1). Grade 1 is considered MHE while grades 2 to 3 reflect severity of OHE manifestations and Grade 4 is reserved for comatose patients. No laboratory test is presently available to diagnose OHE. Although ammonia plays a key role in the pathogenesis of OHE and is often found to be elevated in population studies, on an individual basis serum ammonia level is not accurate in the diagnosis of $\mathrm{OHE}^{28}$

\section{CLINICAL IMPACT}

\section{Clinical outcomes and healthcare utilization}

Overt hepatic encephalopathy has been shown to have a poor prognosis independent of severity of liver disease. One study evaluating hospitalized patients with OHE found that 1 year survival probability was $42 \%$ and $23 \%$ at 3 years. ${ }^{29}$ Similarly past studies have also found 1 year survival to be $20 \%-40 \%$ with 3 year survival of 15\%. ${ }^{30,31}$ A retrospective analysis of patients with severe HE requiring intensive care unit admission found 1 year mortality to be 54\%. ${ }^{32}$ Furthermore patients with severe $\mathrm{HE}$ (grade 3 to 4 ) at time of waitlist registration for liver transplant had a significantly greater risk of 90-day mortal- 
ity when compared to patients without HE (hazard ratio [HR], 1.6). ${ }^{33}$ Significance of MHE on clinical outcomes is still unclear. A recent study by Patidar et al. ${ }^{34}$ measured clinical outcomes of 170 cirrhotic patients several months. Of these patients 56\% had MHE and were found to have a higher risk of developing OHE (HR, 2.1; 95\% confidence interval [CI], 1.01 to 4.5), hospitalization (HR, 2.5; 95\% CI, 1.4 to 4.5) and death/transplant (HR, 3.4; 95\% CI, 1.2 to 9.7$)$.

Resource utilization by patients with HE is currently on the rise. From 2005 to 2009 there was a 55.1\% increase on hospitalizations costs from $\$ 4.677$ billion to $\$ 7.253$ billion in the United States. ${ }^{1}$ Over the same period total national cost increased from $\$ 1.7$ billion to $\$ 2$ billion, a $23.8 \%$ increase once adjusted for inflation. Furthermore the total cost per each HE hospitalization increased from \$22,511 in 2004 to $\$ 37,598$ in 2010 . $^{35}$ Frequent readmission plays a large role in the high healthcare utilization seen in HE patients. A retrospective analysis of data from over 500 U.S. hospitals between 2010 to 2011 showed a readmission rate of $17.6 \%$ in the first 30 days and 39.5\% within 1 year. ${ }^{36}$ Yet another study found readmission rates of $37 \%$ in the first month. ${ }^{37}$ Of these readmission the authors concluded that 25\% could have been prevented with better patient understanding of their medical regiment and closer outpatient follow-up. Hospital readmissions were also associated with higher mortality, independent of disease severity. The same study reported the mean cost of readmission within 1 week to be $\$ 28,898$ and $\$ 20,581$ for readmissions between weeks 1 and 4 .

\section{Disability and burden on caregivers}

The impact of HE on both patients, their care givers, and society as a whole can be profound. Often patients with frequent episodes of HE are left with permanent disabilities and poor quality of life that strain resources of care givers and healthcare systems. Several clinical studies have shown that patients develop cumulative deficits in learning capacity, memory and response inhibition even after resolution of overt OHE. ${ }^{19,20,38}$

Several studies have shown the patients with HE have difficulty operating motor vehicles. Bajaj et al. ${ }^{39}$ followed 167 cirrhotic patients over 1 year and found that patients with MHE had a $22 \%$ rate of traffic violations compared to $3 \%$ in those without MHE. In yet another study 51 chronic cirrhotic patients and 48 age matched controls were subjected to driving tests and assessment by instructors. ${ }^{40}$ Per the final assessments only 39\% of patients with OHE and 48\% of patients with MHE were fit to drive, compared to $75 \%$ in the control group. In patients with OHE the primary difficult was cognitive defects and prolonged reaction times. In the MHE most mistakes were secondary to attention deficits.

Deficits in reaction times and attention in patients with HE can increase the risk of falls. This is particularly dangerous as most HE patients have underlying cirrhosis which often accompanies coagulopathy. Román et al. ${ }^{41}$ evaluated 130 cirrhotic pa- tients and 43 controls for falls over a 12 month period of time. Of the patients with cirrhosis, 45 were diagnosed with MHE and were found to have a higher incidence of falls (40\%) as compared to cirrhotic patients without MHE (12.9\%) or controls (11.6\%).

Due to their significant disabilities and inability to care for themselves, HE patients often put a great burden on caregivers. This can be seen often in clinical practice however there has been little published data on the matter. Bajaj et al. ${ }^{42}$ evaluated 104 patients with cirrhosis, 44\% of which had previous episodes of HE. Patients and caregivers were evaluated in a series of questionnaires. Regarding patient employment, 44\% had stopped working after the diagnosis of cirrhosis although 71\% thought continuing to work was important to them and would do so if they could. Furthermore of the patients that continued to work, 53\% had to decrease their hours. The financial burden of cirrhosis and HE often severely impacts the quality of life in these families. Fifty-six percent of families responded that they had to stop saving to pay for medical costs and $46 \%$ had incurred debt. Overdue bills (15\%), reduced funding for education (16\%), lack of funding for food (11\%), loss of homes (15\%) and bankruptcy (7\%) were also reported. When evaluating caregivers for depression, 18\% had mild depression while 5\% had moderate and 5\% had severe depression. Twenty-two percent of the caregivers had mild anxiety, 12\% had moderate and 5\% had severe anxiety. Interestingly model for end stage liver disease (MELD) and patient's cognitive status showed strong correlations with caregiver burden, measure via Perceived Caregiver Burden Scale and Zarit Burden Interview short form questionnaire.

\section{MANAGEMENT}

The approach to HE depends on its severity (Fig. 3). Treatment of inciting event is the first priority as 90\% of HE patients will have a precipitating factor. ${ }^{43}$ Management of acute mental status changes to return patient back to baseline should be the next priority. Lastly therapy should be directed to prevent recurrence of HE, secondary prophylaxis. Medical treatment for HE has been limited. Common medications include lactulose and rifaximin (Table 1). Nutrition may also play a key role in managing HE and preventing recurrence. ${ }^{44}$ Second line, less accepted therapies, include probiotics, branched chain amino acids (BCAAs), flumazenil, zinc and ammonia scavengers.

\section{Nutrition}

Maintaining adequate nutrition is vital in patients with HE. Protein calorie malnutrition is commonly found in HE patients and has been associated with poor prognosis. ${ }^{45}$ Often inappropriate recommendations to reduce protein intake, frequent body fluid removal via paracentesis or anemia from gastrointestinal bleeding all likely contribute. ${ }^{46}$ Maintaining adequate protein 


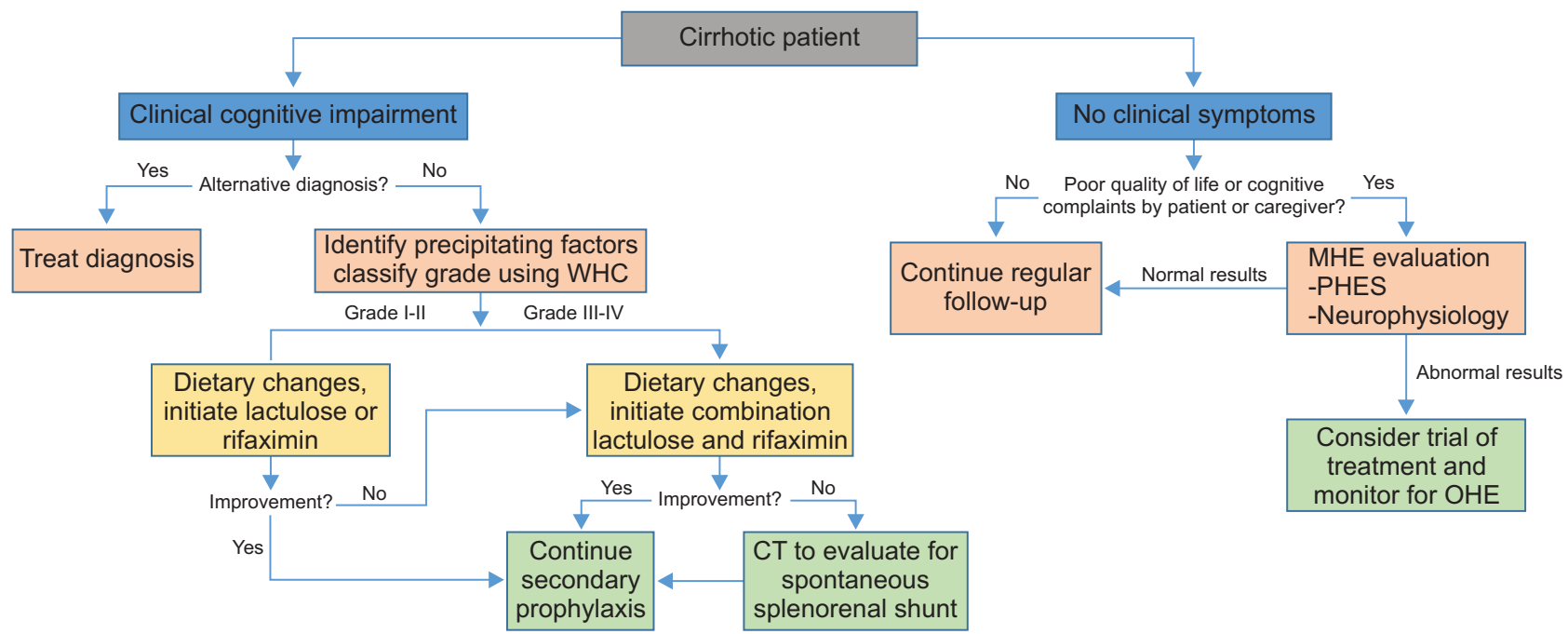

Fig. 3. Evaluation and management of hepatic encephalopathy in cirrhotic patients.

WHC, West Haven Criteria; CT, computed tomography; MHE, minimal hepatic encephalopathy; PHES, Psychometric Hepatic Encephalopathy Score; OHE, overt hepatic encephalopathy.

Table 1. Common Treatment Options for Hepatic Encephalopathy

\begin{tabular}{lll}
\hline Treatment & \multicolumn{1}{c}{ Mechanism } & Comments \\
\hline Lactulose & Prebiotics, laxative, alters gut flora to decrease ammonia production and absorption & First line agent \\
Rifaximin & Nonabsorbable antibiotic & Add-on to lactulose \\
& Alters gut flora thereby decreasing ammonia production & Can be used as first-line \\
\hline
\end{tabular}

intake is essential in preventing muscle wasting. After the liver, skeletal muscle is the largest site of ammonia metabolism. ${ }^{47}$ Similar to astrocytes, skeletal muscles use glutamine synthetase to generate glutamine from ammonia and glutamate. The glutamate is in turn synthetized from branch chain amino acids. This pathways is likely the reason HE patients have been documented to have lower concentrations of serum branch chained amino acids. Recent studies provide evidence for the benefits of protein intake with every meal as well as late night snacks to prevent protein breakdown overnight. ${ }^{48,49}$ Gluud et al. ${ }^{50}$ conducted a meta-analysis of eight trials evaluating the effects of oral BCAAs in HE and concluded that oral branch chain amino acids improved manifestations of HE but no effect on overall mortality or nutrition status was observed.

An expert panel commissioned by the ISHEN have provided detailed recommendations on nutritional management of patients with hepatic enceophalopathy. ${ }^{44}$ The committee concluded that optimal daily energy intake should be 35 to $40 \mathrm{kcal} / \mathrm{kg}$ ideal body weight with daily protein intake of 1.2 to $1.5 \mathrm{~g} / \mathrm{kg}$ ideal body weight and fiber intake of 25 to $45 \mathrm{~g}$ daily. Furthermore meals should small and evenly distributed during the day with a late night snack of complex carbohydrates to minimize protein utilization overnight. Patients should be encouraged to adhere to diets rich in vegetable and dairy protein. If patients are intolerant of dietary protein, BCAA supplementation is an alternative to consider. Multivitamin use can be considered in patients admitted for decompensated cirrhosis with the addition of specific treatments for clinically apparent vitamin deficiencies.

\section{Nonabsorbable disaccharides}

Nonabsorbable disaccharides, lactulose and lactitol, have long been the mainstay of treatment for HE. Guidelines from both the American Association for the Study of Liver Disease (AASLD) and European Association for the Study of Liver Disease both recommend the use of these agents. ${ }^{51}$ Although nonabsorbable disaccharides are the most commonly prescribed medication for $\mathrm{HE}$, a Cochrane review found no statistically significant effect on mortality when comparing placebo or no intervention to nonabsorbable disaccharides. ${ }^{52}$ Furthermore there was no statistically significant difference between lactulose and lactitol on mortality. Nonabsorbable disaccharides had an increased risk of no improvement when compared to antibiotics. Sharma et al..$^{53}$ conducted an observational study of 231 patients admitted for HE diagnosed by West Haven Criteria and found that 78\% responded to lactulose within 10 days of admission. Response was defined as no longer meeting criteria for HE. Multivariate analysis of baseline characteristics found that total leukocyte count, MELD mean arterial pressure and hepatocellular carcinoma were independent predictors of nonresponse to lactulose. Ap- 
Table 2. Barriers to Lactulose Adherence

\begin{tabular}{ll}
\multicolumn{1}{c}{ Barrier } & \multicolumn{1}{c}{ Comments } \\
\hline Adverse effects & Diarrhea/flatulence, abdominal pain/cramping, nausea, anorexia \\
Lack of understanding & Patients frequently do not understand titrating to 2-3 bowel movements a day \\
Overuse & Leads to dehydration and hyponatremia leading to worsening of hepatic encephalopathy \\
\hline
\end{tabular}

propriate dosing is vital in the effective management of HE. One study showed that nearly 50\% of HE recurrence were related to either no adherence or inappropriate dosing. ${ }^{54}$ Multiple factors contribute to this lack of proper adherence (Table 2). Several studies have shown benefit in primary prophylaxis with lactulose in patients who have never had an episode of HE. ${ }^{55,56}$ Bajaj et al. ${ }^{56}$ showed that treating minimal hepatic with lactulose was cost effective in preventing motor vehicle accidents. An open label, randomized control trial evaluating the use of lactulose in cirrhotic patient who have never had an episode of OHE found that, over the 12 months of follow-up, 11\% of patients in the lactulose group developed symptoms of OHE compared to $28 \%$ in the group that did not receive lactulose. ${ }^{55}$ Current guidelines however do not recommend the routine use of lactulose for primary prophylaxis in HE.

\section{Antibiotics}

Neomycin, vancomycin and metronidazole have been historically used in the setting of $\mathrm{HE}^{27}$ However rifaximin has become the antibiotic of choice in the treatment of HE due to its safety, efficacy and tolerability. In addition rifaximin is nonabsorbable allowing it to concentrate in the gut and limit its systemic absorption. A double-blind, randomized, placebo-controlled trial involving 299 patients in remission from HE found that rifaximin was more effective than placebo in preventing breakthrough HE. ${ }^{57}$ Patients on rifaximin had a reduced risk of an episode of HE as compared to the placebo group with a breakthrough rate of $22.1 \%$ compared to $45.9 \%$ in the placebo group, HR of 0.42 (95\% CI, 0.28 to $0.64 ; \mathrm{p}<0.001$ ). Furthermore patients in the rifaximin group had a HE related hospitalization rate of $13.6 \%$ as compared to $22.6 \%$ in the placebo group, a HR of 0.5 (95\% CI, 0.29 to $0.87 ; p=0.01$ ). The study further evaluated the safety and tolerability of continuous rifaximin used for 24 months and found no increase in adverse events, infection with Clostridium difficile or development of bacterial antibiotic resistance. ${ }^{58}$ Current guidelines from AASLD recommend lactulose as initial treatment for HE, however rifaximin monotherapy has been shown to be effective. ${ }^{51,59}$ Furthermore while combination therapy for low grade HE has not been shown to improve outcomes, combination therapy should be considered for recurrent HE on lactulose or severe HE (Fig. 2). ${ }^{60,61}$

When compared to neomycin, rifaximin was to be at least as effective in reducing blood ammonia levels and had less adverse effects. ${ }^{62}$ Furthermore rifaximin has been shown to be noninferior to lactulose. ${ }^{63}$ Combination treatment with rifaximin and lactulose is likely more beneficial than lactulose alone. Sharma et al. conducted a randomized, double-blind, placebo-controlled trial evaluating lactulose plus rifaximin compared to lactulose alone and showed that combination therapy was indeed more effective. ${ }^{64}$ Of the patients treated with combination therapy, $76 \%$ had complete reversal of HE as compared to 50.8\% in the lactulose only group. Furthermore there was significant improvements in mortality and hospital stay in the combination group.

Neomycin has been previously used extensively in the management of HE. It has been shown to reduce the intestinal production of ammonia from glutamine and likely acts as a glutaminase inhibitor. ${ }^{65}$ However adverse effects such as ototoxicity and nephrotoxicity limits its use in clinical practice. ${ }^{61}$ Likewise, metronidazole is not currently recommended due to concern of nephrotoxicity and neurotoxicity with long-term use. ${ }^{66}$ Likewise vancomycin is nephrotoxic and extensive use increases the risk of renal injury as well as development of resistance. ${ }^{61}$

\section{Probiotics}

As gut bacteria play a central role in producing ammonia it has been theorized that altering gut flora using probiotics may be beneficial in HE. Several randomized control trials have been conducted comparing probiotics to placebo or no treatment as well as lactulose and have shown some benefit. ${ }^{67-69}$ The use of probiotics in secondary prophylaxis was evaluated in an openlabel, randomized controlled trial involving 235 patients divided into a lactulose, probiotic or no therapy arm. ${ }^{70}$ Recurrence rate was found to be $26.2 \%$ (18 of 68) in the lactulose arm, 34.4\% (22 of 64) in the probiotic arm and 56.9\% (37 of 68) in the no therapy arm. While both lactulose and probiotics were significantly more effective than no therapy, no significant difference was found between the two. Zhao et al. ${ }^{71}$ recently conducted a systemic review of nine randomized control trials and concluded that probiotics were associated with improvement in MHE, prophylaxis of $\mathrm{OHE}$ and reduction in severe adverse events.

\section{Polyethylene glycol}

Prior to the widespread adoption of nonabsorbable disaccharides, simple laxatives were often used to treat HE with some benefit suggesting that bowel evacuation alone may effectively treat HE. ${ }^{72}$ Polyethylene glycol is a commonly used, safe and highly effective laxative that has recently been proposed as a possible agent for HE. Rahimi et al. ${ }^{73}$ compared the use of polyethylene glycol 3350-electrolyte solution versus lactulose in the 
treatment in 50 patients with underlying cirrhosis admitted for HE. Primary endpoint measured was improvement of HE grade by 1 or more points determined by the HE scoring algorithm. Of the patients who received polyethylene glycol, 21 of 23 (91\%) had an improvement in HE as compared to 13 of 25 (52\%) in the lactulose arm, a significant difference $(p<0.01)$. Furthermore the median time for HE resolution in the polyethylene glycol group was 1 day as compared to 2 days in the standard therapy $\operatorname{arm}(\mathrm{p}=0.01)$.

\section{Flumazenil}

Flumazenil is a benzodiazepine antagonist that targets GABA receptors. Laccetti et al. ${ }^{74}$ conducted a double blind randomized, placebo controlled trial evaluating the use of flumazenil in acute HE. Of the patients that were treated with flumazenil 79\% (22 of 28) showed clinical improvement compared to 54\% (14 of 26) in the placebo arm. No mortality benefit was observed.

\section{Ammonia scavengers}

Ammonia scavengers help to increase ammonia clearance and thus reduce systemic concentrations of ammonia. Glycerol phenylbutyrate lowers serum ammonia by providing an alternative pathway for renal clearance of nitrogen via phenylacetylglutamine. A recent phase II clinical trial showed that glycerol phenylbutyrate reduced HE events (21\% vs 36\% in placebo group, $p=0.02$ ), was associated with fewer HE hospitalizations and lowered plasma ammonia. ${ }^{75}$ In subgroup analysis of patients taking lactulose at baseline, the glycerol phenylbutyrate group had a $\mathrm{HE}$ event rate of $22 \%$ versus $45 \%$ in the placebo group $(p<0.01)$. However there was no difference between treatment arms in those patients taking rifaximin at baseline. Ornithine pheynlacetate is another ammonia scavenger that augments the same pathway as glycerol phenylbutyrate, leading to excretion of ammonia in urine as pheynylacetylglutamine. Ornithine phenylacetate has been shown to reduce plasma ammonia levels in patients with decompensated cirrhosis. ${ }^{76}$ Ornithine and aspartate are substrates of the urea cycle.

\section{L-ornithine I aspartate}

L-ornithine 1 aspartate (LOLA) seem to stimulate enzyme activity in residual hepatocytes by increasing carbamyl phosphate synthetase in periportal hepatocytes and increasing glutamine synthase in pericentral hepatocytes, leading to increased urea excretion. ${ }^{77}$ A systematic review of eight randomized control trials evaluating the effectiveness of LOLA showed that LOLA significantly improved HE when compared to placebo or no intervention. ${ }^{78}$ Similarly LOLA had a significantly greater reduction in plasma ammonia levels when compared to placebo or no intervention. When compared to lactulose, LOLA demonstrated similar improvement in HE with no statistically significant difference.

\section{Zinc}

Studies have shown that low zinc is associated with impairment of urea cycle enzymes leading to elevated ammonia levels. ${ }^{79}$ Chavez-Tapia et al. ${ }^{80}$ conducted a systemic review of four trials to evaluate the use of zinc in $\mathrm{HE}$ and found that oral zinc supplementation was associated with significant improvement in psychometric tests but did not affect recurrence rates. However no evidence on other clinical outcomes is currently available.

\section{Portosystemic shunts}

Medically refractory HE should raise suspicion of a spontaneous splenorenal shunt. One study evaluated the use of balloonoccluded retrograde transvenous obliteration of large spontaneous splenorenal shunts in patients with severe recurrent HE with the authors reporting symptomatic improvement of all patients at the time of discharge as well as at 4 month followup. ${ }^{81}$ Furthermore a retrospective study evaluating patients with recurrent HE who achieved complete occlusion of portosystemic shunts compared to patients who did not undergo embolization showed some benefit in embolization. ${ }^{82}$ Subgroup analyses of patients with MELD <15 and no hepatocellular carcinoma showed that 2-year survival was significantly higher in the embolization group than the control group. Median changed in MELD, Child-Turcotte-Pugh scores and liver volumes also favored the embolization group. Laleman et al..$^{83}$ conducted another retrospective, multicenter analysis evaluating the efficacy and safety with embolization of large spontaneous portosystemic shunts for treatment of chronic therapy-refractory HE. Thirty seven patients were included in the study. When evaluating for recurrence within 100 days post embolization, 22 of 37 patients (59\%) were symptom-free, a significant difference from prior to embolization $(\mathrm{p}<0.001)$. Eighteen patients continued to be symptom free over a mean follow-up period of $691 \pm 157$ days, again a significant difference from prior to embolization $(\mathrm{p}<0.001)$.

Similarly, HE is often associated with iatrogenic shunt placement, such as TIPS. It is estimated that 5\% to $35 \%$ of patients who undergo TIPS develop new or worsened HE post procedure. ${ }^{84}$ Furthermore 3\% to $7 \%$ of patients develop HE that is refractory to medical therapy, requiring shunt modification or emergent liver transplantation. ${ }^{85}$ Bare metal stents are often associated with frequent HE post procedure with decreased frequency over time. This phenomenon is likely due to the development of shunt stenosis that progressively reduces the amount of shunted blood. ${ }^{86,87} \mathrm{New}$ polyterafluroethylene-covered endoprostheses have reduced the number of shunt stenoses. Interestingly studies have shown that HE in patients with covered stents is either reduced or unchanged. ${ }^{88,89}$ This observation may be due to the fact that covered stent patients often require less dilations and restenting thereby reducing the episodes of 
HE that may occur right after shunt revisions. ${ }^{90} \mathrm{~A}$ major risk factor for HE after TIPS is having recurrent HE prior to undergoing TIPS. This should be considered a contraindication for TIPS. ${ }^{91}$ Other risk factors include age, low portacaval pressure gradient, high Child-Pugh scores, high creatinine, low serum sodium concentrations and MHE prior to TIPS. ${ }^{87,92-94}$ In cases of chronic refractory HE post TIPS, shunt revision is an option. Fanelli et al. ${ }^{95}$ conducted a retrospective analysis of 12 patients who underwent reduction of TIPS due to severe HE refractory to medical intervention. Symptoms of HE disappeared in 18 to 26 hours post procedure in all patients. There was no recurrence at a mean follow-up of $73.9( \pm 61.88)$ weeks. However shunt revisions may result in increased portal hypertension and subsequently worsening varices or refractory ascites. ${ }^{90}$ Thus careful consideration should be made in regard to which patients qualify for TIPS and which patients with recurrent HE post TIPS should undergo revision.

\section{Artificial liver support and liver transplant}

Albumin dialysis with molecular adsorbent recirculating system (MARS) decreases plasma concentrations of bilirubin, ammonia and creatinine as well as improves portal pressures and HE in patients with acute-on-chronic liver failure. ${ }^{96-98}$ However a recent study found no survival benefit of MARS therapy in acute-on-chronic liver failure patient, although there was a nonsignificant improvement in severe HE. ${ }^{99}$ Recurrent OHE in setting of liver failure is an indication for liver transplant and liver transplant remains the only definitive treatment for HE. However a diagnosis of HE does not increase your likelihood of receiving a liver transplant.

\section{CONCLUSIONS}

Hepatic encephalopathy is an important complication of liver cirrhosis associated with morbidity and mortality. Hepatic encephalopathy is also associated with substantial health care utilization and adverse impact of care givers. Early recognition of HE is essential for timely management. Treatment of HE revolves around its severity and includes lactulose and rifaximin either alone or in combination. Nutritional modification may also improve HE recurrence. Investigation is still ongoing regarding options for treating medically refractory HE including spontaneous splenorenal shunt embolization, glycerol phenylbutyrate, and artificial liver support.

\section{CONFLICTS OF INTEREST}

V.S. is on the speakers bureau for Salix pharmaceuticals. No potential conflict of interest relevant to this article was reported.

\section{REFERENCES}

1. Stepanova M, Mishra A, Venkatesan C, Younossi ZM. In-hospital mortality and economic burden associated with hepatic encephalopathy in the United States from 2005 to 2009. Clin Gastroenterol Hepatol 2012;10:1034-1041.e1.

2. Parekh PJ, Balart LA. Ammonia and its role in the pathogenesis of hepatic encephalopathy. Clin Liver Dis 2015;19:529-537.

3. Poh Z, Chang PE. A current review of the diagnostic and treatment strategies of hepatic encephalopathy. Int J Hepatol 2012;2012:480309.

4. Brusilow SW. Hyperammonemic encephalopathy. Medicine (Baltimore) 2002;81:240-249.

5. Häussinger D, Kircheis G, Fischer R, Schliess F, vom Dahl S. Hepatic encephalopathy in chronic liver disease: a clinical manifestation of astrocyte swelling and low-grade cerebral edema? J Hepatol 2000;32:1035-1038.

6. Zhang XD, Zhang LJ, Wu SY, Lu GM. Multimodality magnetic resonance imaging in hepatic encephalopathy: an update. World J Gastroenterol 2014;20:11262-11272.

7. Häussinger D, Schliess F. Pathogenetic mechanisms of hepatic encephalopathy. Gut 2008;57:1156-1165.

8. Reinehr R, Görg B, Becker S, et al. Hypoosmotic swelling and ammonia increase oxidative stress by NADPH oxidase in cultured astrocytes and vital brain slices. Glia 2007;55:758-771.

9. Albrecht J, Norenberg MD. Glutamine: a Trojan horse in ammonia neurotoxicity. Hepatology 2006;44:788-794.

10. Görg B, Qvartskhava N, Bidmon HJ, et al. Oxidative stress markers in the brain of patients with cirrhosis and hepatic encephalopathy. Hepatology 2010;52:256-265.

11. Ahboucha S, Talani G, Fanutza T, et al. Reduced brain levels of DHEAS in hepatic coma patients: significance for increased GABAergic tone in hepatic encephalopathy. Neurochem Int 2012;61:48-53.

12. Sergeeva OA. GABAergic transmission in hepatic encephalopathy. Arch Biochem Biophys 2013;536:122-130.

13. Jones EA. Ammonia, the GABA neurotransmitter system, and hepatic encephalopathy. Metab Brain Dis 2002;17:275-281.

14. Kerlan RK Jr, LaBerge JM, Baker EL, et al. Successful reversal of hepatic encephalopathy with intentional occlusion of transjugular intrahepatic portosystemic shunts. J Vasc Interv Radiol 1995;6:917-921.

15. McCrea M, Cordoba J, Vessey G, Blei AT, Randolph C. Neuropsychological characterization and detection of subclinical hepatic encephalopathy. Arch Neurol 1996;53:758-763.

16. Weissenborn K, Bokemeyer M, Krause J, Ennen J, Ahl B. Neurological and neuropsychiatric syndromes associated with liver disease. AIDS 2005;19 Suppl 3:S93-S98.

17. Montagnese S, De Pittà C, De Rui M, et al. Sleep-wake abnormalities in patients with cirrhosis. Hepatology 2014;59:705-712.

18. Bajaj JS, Wade JB, Sanyal AJ. Spectrum of neurocognitive impairment in cirrhosis: implications for the assessment of hepatic 
encephalopathy. Hepatology 2009;50:2014-2021.

19. Bajaj JS, Schubert CM, Heuman DM, et al. Persistence of cognitive impairment after resolution of overt hepatic encephalopathy. Gastroenterology 2010;138:2332-2340.

20. Umapathy S, Dhiman RK, Grover S, Duseja A, Chawla YK. Persistence of cognitive impairment after resolution of overt hepatic encephalopathy. Am J Gastroenterol 2014;109:1011-1019.

21. Bajaj JS. Review article: the modern management of hepatic encephalopathy. Aliment Pharmacol Ther 2010;31:537-547.

22. Mardini H, Saxby BK, Record CO. Computerized psychometric testing in minimal encephalopathy and modulation by nitrogen challenge and liver transplant. Gastroenterology 2008;135:15821590.

23. Bajaj JS, Heuman DM, Sterling RK, et al. Validation of encephalapp, smartphone-based stroop test, for the diagnosis of covert hepatic encephalopathy. Clin Gastroenterol Hepatol 2015;13:1828-1835.e1.

24. Sharma P, Sharma BC, Sarin SK. Critical flicker frequency for diagnosis and assessment of recovery from minimal hepatic encephalopathy in patients with cirrhosis. Hepatobiliary Pancreat Dis Int 2010;9:27-32.

25. Montagnese S, Amodio P, Morgan MY. Methods for diagnosing hepatic encephalopathy in patients with cirrhosis: a multidimensional approach. Metab Brain Dis 2004;19:281-312.

26. Bajaj JS, Cordoba J, Mullen KD, et al. Review article. The design of clinical trials in hepatic encephalopathy: an International Society for Hepatic Encephalopathy and Nitrogen Metabolism (ISHEN) consensus statement. Aliment Pharmacol Ther 2011;33:739-747.

27. Conn HO, Leevy CM, Vlahcevic ZR, et al. Comparison of lactulose and neomycin in the treatment of chronic portal-systemic encephalopathy: a double blind controlled trial. Gastroenterology 1977;72(4 Pt 1):573-583.

28. Ge PS, Runyon BA. Serum ammonia level for the evaluation of hepatic encephalopathy. JAMA 2014;312:643-644.

29. Bustamante J, Rimola A, Ventura PJ, et al. Prognostic significance of hepatic encephalopathy in patients with cirrhosis. $\mathrm{J}$ Hepatol 1999;30:890-895.

30. Christensen E, Krintel JJ, Hansen SM, Johansen JK, Juhl E. Prognosis after the first episode of gastrointestinal bleeding or coma in cirrhosis: survival and prognostic factors. Scand J Gastroenterol 1989;24:999-1006.

31. Saunders JB, Walters JR, Davies AP, Paton A. A 20-year prospective study of cirrhosis. Br Med J (Clin Res Ed) 1981;282:263-266.

32. Fichet J, Mercier E, Genée 0, et al. Prognosis and 1-year mortality of intensive care unit patients with severe hepatic encephalopathy. J Crit Care 2009;24:364-370.

33. Wong RJ, Gish RG, Ahmed A. Hepatic encephalopathy is associated with significantly increased mortality among patients awaiting liver transplantation. Liver Transpl 2014;20:1454-1461.

34. Patidar KR, Thacker LR, Wade JB, et al. Covert hepatic encephalopathy is independently associated with poor survival and increased risk of hospitalization. Am J Gastroenterol
2014;109:1757-1763.

35. Neff GW, Kemmer N, Duncan C, Alsina A. Update on the management of cirrhosis: focus on cost-effective preventative strategies. Clinicoecon Outcomes Res 2013;5:143-152.

36. Saab S. Evaluation of the impact of rehospitalization in the management of hepatic encephalopathy. Int J Gen Med 2015;8:165173.

37. Volk ML, Tocco RS, Bazick J, Rakoski MO, Lok AS. Hospital readmissions among patients with decompensated cirrhosis. Am J Gastroenterol 2012;107:247-252.

38. Riggio 0, Ridola L, Pasquale C, et al. Evidence of persistent cognitive impairment after resolution of overt hepatic encephalopathy. Clin Gastroenterol Hepatol 2011;9:181-183.

39. Bajaj JS, Saeian K, Schubert CM, et al. Minimal hepatic encephalopathy is associated with motor vehicle crashes: the reality beyond the driving test. Hepatology 2009;50:1175-1183.

40. Kircheis G, Knoche A, Hilger N, et al. Hepatic encephalopathy and fitness to drive. Gastroenterology 2009;137:1706-1715.e9.

41. Román E, Córdoba J, Torrens M, et al. Minimal hepatic encephalopathy is associated with falls. Am J Gastroenterol 2011;106:476-482.

42. Bajaj JS, Wade JB, Gibson DP, et al. The multi-dimensional burden of cirrhosis and hepatic encephalopathy on patients and caregivers. Am J Gastroenterol 2011;106:1646-1653.

43. Strauss E, Tramote R, Silva EP, et al. Double-blind randomized clinical trial comparing neomycin and placebo in the treatment of exogenous hepatic encephalopathy. Hepatogastroenterology 1992;39:542-545.

44. Amodio P, Bemeur C, Butterworth R, et al. The nutritional management of hepatic encephalopathy in patients with cirrhosis: International Society for Hepatic Encephalopathy and Nitrogen Metabolism Consensus. Hepatology 2013;58:325-336.

45. Chadalavada R, Sappati Biyyani RS, Maxwell J, Mullen K. Nutrition in hepatic encephalopathy. Nutr Clin Pract 2010;25:257-264.

46. Bémeur C, Desjardins P, Butterworth RF. Role of nutrition in the management of hepatic encephalopathy in end-stage liver failure. J Nutr Metab 2010;2010:489823.

47. Holecek M. Branched-chain amino acids and ammonia metabolism in liver disease: therapeutic implications. Nutrition 2013;29:1186-1191.

48. Holecek M. Ammonia and amino acid profiles in liver cirrhosis: effects of variables leading to hepatic encephalopathy. Nutrition 2015;31:14-20.

49. Tsien CD, McCullough AJ, Dasarathy S. Late evening snack: exploiting a period of anabolic opportunity in cirrhosis. J Gastroenterol Hepatol 2012;27:430-441.

50. Gluud LL, Dam G, Borre M, et al. Oral branched-chain amino acids have a beneficial effect on manifestations of hepatic encephalopathy in a systematic review with meta-analyses of randomized controlled trials. J Nutr 2013;143:1263-1268.

51. Vilstrup H, Amodio P, Bajaj J, et al. Hepatic encephalopathy in chronic liver disease: 2014 Practice Guideline by the American 
Association for the Study of Liver Diseases and the European Association for the Study of the Liver. Hepatology 2014;60:715735.

52. Als-Nielsen B, Gluud LL, Gluud C. Nonabsorbable disaccharides for hepatic encephalopathy. Cochrane Database Syst Rev 2004;2:CD003044.

53. Sharma P, Sharma BC, Sarin SK. Predictors of nonresponse to lactulose in patients with cirrhosis and hepatic encephalopathy. Eur J Gastroenterol Hepatol 2010;22:526-531.

54. Bajaj JS, Sanyal AJ, Bell D, Gilles H, Heuman DM. Predictors of the recurrence of hepatic encephalopathy in lactulose-treated patients. Aliment Pharmacol Ther 2010;31:1012-1017.

55. Sharma P, Sharma BC, Agrawal A, Sarin SK. Primary prophylaxis of overt hepatic encephalopathy in patients with cirrhosis: an open labeled randomized controlled trial of lactulose versus no lactulose. J Gastroenterol Hepatol 2012;27:1329-1335.

56. Bajaj JS, Pinkerton SD, Sanyal AJ, Heuman DM. Diagnosis and treatment of minimal hepatic encephalopathy to prevent motor vehicle accidents: a cost-effectiveness analysis. Hepatology 2012;55:1164-1171.

57. Bass NM, Mullen KD, Sanyal A, et al. Rifaximin treatment in hepatic encephalopathy. N Engl J Med 2010;362:1071-1081.

58. Mullen KD, Sanyal AJ, Bass NM, et al. Rifaximin is safe and well tolerated for long-term maintenance of remission from overt hepatic encephalopathy. Clin Gastroenterol Hepatol 2014;12:13901397.e2.

59. Neff GW, Jones M, Broda T, et al. Durability of rifaximin response in hepatic encephalopathy. J Clin Gastroenterol 2012;46:168-171.

60. Mohammad RA, Regal RE, Alaniz C. Combination therapy for the treatment and prevention of hepatic encephalopathy. Ann Pharmacother 2012;46:1559-1563.

61. Leise MD, Poterucha JJ, Kamath PS, Kim WR. Management of hepatic encephalopathy in the hospital. Mayo Clin Proc 2014;89:241-253.

62. Pedretti G, Calzetti C, Missale G, Fiaccadori F. Rifaximin versus neomycin on hyperammoniemia in chronic portal systemic encephalopathy of cirrhotics: a double-blind, randomized trial. Ital J Gastroenterol 1991;23:175-178.

63. Jiang $Q$, Jiang XH, Zheng MH, Jiang LM, Chen YP, Wang L. Rifaximin versus nonabsorbable disaccharides in the management of hepatic encephalopathy: a meta-analysis. Eur J Gastroenterol Hepatol 2008;20:1064-1070.

64. Sharma BC, Sharma P, Lunia MK, Srivastava S, Goyal R, Sarin SK. A randomized, double-blind, controlled trial comparing rifaximin plus lactulose with lactulose alone in treatment of overt hepatic encephalopathy. Am J Gastroenterol 2013;108:14581463.

65. Hawkins RA, Jessy J, Mans AM, Chedid A, DeJoseph MR. Neomycin reduces the intestinal production of ammonia from glutamine. Adv Exp Med Biol 1994;368:125-134.

66. Morgan MH, Read AE, Speller DC. Treatment of hepatic encephalopathy with metronidazole. Gut 1982;23:1-7.
67. Shavakhi A, Hashemi H, Tabesh E, et al. Multistrain probiotic and lactulose in the treatment of minimal hepatic encephalopathy. J Res Med Sci 2014;19:703-708.

68. Dhiman RK, Rana B, Agrawal S, et al. Probiotic VSL\#3 reduces liver disease severity and hospitalization in patients with cirrhosis: a randomized, controlled trial. Gastroenterology 2014;147:13271337.e3.

69. Lunia MK, Sharma BC, Sharma P, Sachdeva S, Srivastava S. Probiotics prevent hepatic encephalopathy in patients with cirrhosis: a randomized controlled trial. Clin Gastroenterol Hepatol 2014;12:1003-1008.e1.

70. Agrawal A, Sharma BC, Sharma P, Sarin SK. Secondary prophylaxis of hepatic encephalopathy in cirrhosis: an open-label, randomized controlled trial of lactulose, probiotics, and no therapy. Am J Gastroenterol 2012;107:1043-1050.

71. Zhao LN, Yu T, Lan SY, et al. Probiotics can improve the clinical outcomes of hepatic encephalopathy: an update meta-analysis. Clin Res Hepatol Gastroenterol 2015;39:674-682.

72. Manning RT, Delp M. Management of hepatocerebral intoxication. N Engl J Med 1958;258:55-62.

73. Rahimi RS, Singal AG, Cuthbert JA, Rockey DC. Lactulose vs polyethylene glycol 3350: electrolyte solution for treatment of overt hepatic encephalopathy. The HELP randomized clinical trial. JAMA Intern Med 2014;174:1727-1733.

74. Laccetti M, Manes G, Uomo G, Lioniello M, Rabitti PG, Balzano A. Flumazenil in the treatment of acute hepatic encephalopathy in cirrhotic patients: a double blind randomized placebo controlled study. Dig Liver Dis 2000;32:335-338.

75. Rockey DC, Vierling JM, Mantry P, et al. Randomized, doubleblind, controlled study of glycerol phenylbutyrate in hepatic encephalopathy. Hepatology 2014;59:1073-1083.

76. Ventura-Cots M, Arranz JA, Simón-Talero M, et al. Safety of ornithine phenylacetate in cirrhotic decompensated patients: an open-label, dose-escalating, single-cohort study. J Clin Gastroenterol 2013;47:881-887.

77. Jover-Cobos M, Khetan V, Jalan R. Treatment of hyperammonemia in liver failure. Curr Opin Clin Nutr Metab Care 2014;17:105110 .

78. Bai M, Yang Z, Qi X, Fan D, Han G. l-ornithine-l-aspartate for hepatic encephalopathy in patients with cirrhosis: a metaanalysis of randomized controlled trials. J Gastroenterol Hepatol 2013;28:783-792.

79. Sundaram V, Shaikh OS. Hepatic encephalopathy: pathophysiology and emerging therapies. Med Clin North Am 2009;93:819836.

80. Chavez-Tapia NC, Cesar-Arce A, Barrientos-Gutiérrez T, VillegasLópez FA, Méndez-Sanchez N, Uribe M. A systematic review and meta-analysis of the use of oral zinc in the treatment of hepatic encephalopathy. Nutr J 2013;12:74.

81. Mukund A, Rajesh S, Arora A, Patidar Y, Jain D, Sarin SK. Efficacy of balloon-occluded retrograde transvenous obliteration of large spontaneous lienorenal shunt in patients with severe 
recurrent hepatic encephalopathy with foam sclerotherapy: initial experience. J Vasc Interv Radiol 2012;23:1200-1206.

82. An J, Kim KW, Han S, Lee J, Lim YS. Improvement in survival associated with embolisation of spontaneous portosystemic shunt in patients with recurrent hepatic encephalopathy. Aliment Pharmacol Ther 2014;39:1418-1426.

83. Laleman W, Simon-Talero M, Maleux G, et al. Embolization of large spontaneous portosystemic shunts for refractory hepatic encephalopathy: a multicenter survey on safety and efficacy. Hepatology 2013;57:2448-2457.

84. Zuckerman DA, Darcy MD, Bocchini TP, Hildebolt CF. Encephalopathy after transjugular intrahepatic portosystemic shunting: analysis of incidence and potential risk factors. AJR Am J Roentgenol 1997;169:1727-1731.

85. Forauer AR, McLean GK. Transjugular intrahepatic portosystemic shunt constraining stent for the treatment of refractory postprocedural encephalopathy: a simple design utilizing a Palmaz stent and Wallstent. J Vasc Interv Radiol 1998;9:443-446.

86. Sanyal AJ, Freedman AM, Shiffman ML, Purdum PP 3rd, Luketic VA, Cheatham AK. Portosystemic encephalopathy after transjugular intrahepatic portosystemic shunt: results of a prospective controlled study. Hepatology 1994;20(1 Pt 1):46-55.

87. Riggio 0, Merlli M, Pedretti G, et al. Hepatic encephalopathy after transjugular intrahepatic portosystemic shunt: incidence and risk factors. Dig Dis Sci 1996;41:578-584.

88. Bureau C, Garcia-Pagan JC, Otal P, et al. Improved clinical outcome using polytetrafluoroethylene-coated stents for TIPS: results of a randomized study. Gastroenterology 2004;126:469-475.

89. Yang Z, Han G, Wu Q, et al. Patency and clinical outcomes of transjugular intrahepatic portosystemic shunt with polytetrafluoroethylene-covered stents versus bare stents: a meta-analysis. J Gastroenterol Hepatol 2010;25:1718-1725.

90. Riggio 0, Nardelli S, Moscucci F, Pasquale C, Ridola L, Merli M. Hepatic encephalopathy after transjugular intrahepatic portosystemic shunt. Clin Liver Dis 2012;16:133-146.

91. Riggio 0, Ridola L, Lucidi C, Angeloni S. Emerging issues in the use of transjugular intrahepatic portosystemic shunt (TIPS) for management of portal hypertension: time to update the guidelines? Dig Liver Dis 2010;42:462-467.

92. Bai M, Qi X, Yang Z, et al. Predictors of hepatic encephalopathy after transjugular intrahepatic portosystemic shunt in cirrhotic patients: a systematic review. J Gastroenterol Hepatol 2011;26:943-951.

93. Guevara M, Baccaro ME, Ríos J, et al. Risk factors for hepatic encephalopathy in patients with cirrhosis and refractory ascites: relevance of serum sodium concentration. Liver Int 2010;30:11371142.

94. Riggio 0, Masini A, Efrati C, et al. Pharmacological prophylaxis of hepatic encephalopathy after transjugular intrahepatic portosystemic shunt: a randomized controlled study. J Hepatol 2005;42:674-679.

95. Fanelli F, Salvatori FM, Rabuffi P, et al. Management of refractory hepatic encephalopathy after insertion of TIPS: longterm results of shunt reduction with hourglass-shaped balloonexpandable stent-graft. AJR Am J Roentgenol 2009;193:16961702.

96. Catalina MV, Barrio J, Anaya F, et al. Hepatic and systemic haemodynamic changes after MARS in patients with acute on chronic liver failure. Liver Int 2003;23 Suppl 3:39-43.

97. Sen S, Mookerjee RP, Cheshire LM, Davies NA, Williams R, Jalan R. Albumin dialysis reduces portal pressure acutely in patients with severe alcoholic hepatitis. J Hepatol 2005;43:142-148.

98. Laleman W, Wilmer A, Evenepoel P, et al. Effect of the molecular adsorbent recirculating system and Prometheus devices on systemic haemodynamics and vasoactive agents in patients with acute-on-chronic alcoholic liver failure. Crit Care 2006;10:R108

99. Bañares R, Nevens F, Larsen FS, et al. Extracorporeal albumin dialysis with the molecular adsorbent recirculating system in acute-on-chronic liver failure: the RELIEF trial. Hepatology 2013;57:1153-1162.

100. Amodio P. Hepatic encephalopathy. In: Lee SS, Moreau R, eds. Cirrhosis: a practical guide to management. Chichester: John Wiley \& Sons, 2015:105-123. 